\title{
The Research Of Network Coding In D2d Communication System
}

\author{
Zhiyong Zhao, Shixiang Shao, Jun Sun \\ Jiangsu provincial Key Laboratory of Wireless Communication, Nanjing University of Post and Telecommunications \\ Nanjing, 210003, China \\ Email: zzy900106@163.com \{shaosx, sunjun\}@njupt.edu.cn
}

\begin{abstract}
D2D (Device-to-Device) communications is the hotspot of next generation of communications technology. There is a challenge that how to decrease the interference between cellular user and D2D user. Unlike resource allocation and power control, the paper proposes a new method that is network coding to reduce the interference between the cellular user and the D2D user. Adding a relay in a D2D pair and use network coding to received data stream, then broadcast to D2D pair to complete information exchange. Such programs procedures can improve the D2D communication system performance, which can be demonstrated through qualitative analysis and extensive simulation.

Index Terms- D2D; Network coding; system capacity; outage probability
\end{abstract}

\section{Introduction}

With the development of era, the demand for communication quality is rising. Traditional cellular communication due to the problem of area coverage and system capacity unable to meet the needs and requirements of people communicate. So the next generation standard LTEAdvance proposes to use D2D (Device-to-Device) communication to assist cellular communication. D2D communication is the terminal straight technology, which means that neighbouring terminals can exchange information directly without the help of base station. The papers [1-3] detail discussed the D2D communication application in the next LTE. Studies have shown that the D2D technology has huge advantages in save resources and improve system capacity. Because there is no need for the base station to transmission data, it doesn't need to allocate resources individually and can reuse resource with cellular users, so D2D communication can enhance the utilization the resources of the cellular system. Unlike the conventional short-range communication technology such as Bluetooth and Wi-Fi are used in nonauthorized band, D2D technology specifically refers to the technology used in the license band resource. Although the structure of traditional peer-to-peer network such as Ad-Hoc network has a great deal of flexibility and scalability, but its architecture is sheer distributed, so the network is difficult to control interference and not suitable for the high speed communication. What's more, the terminal can only get the desired resources through competition with others, which results the low usage of resources. Compared with the Ad-Hoc network, D2D communication can control by the base station, which can significantly improve the resource utilization and optimization interference. The characteristics of D2D technology is short-distance communication and direct communication and it make D2D communication has other advantages, short-distance communication mode can adjust high-rate and low latency communication, direct communication can provide local data sharing service. D2D communication also can use the large number of terminal to expand the coverage of the network.

Even though D2D communication can enhance cellular system performance, it still exist many problems. Because D2D user shares the same resources with a cellular user, it will be make interference between cellular user and D2D user; it will cause degradation in communication quality. How to coordinate and control the interference has become a hot issue in the research of D2D communication. The paper [4] analysis the interference affect to D2D communication. The paper [5] introduces the situations that D2D multiplexes the UL and DL resource of user respectively to control interference. The paper [6] proposes reasonable resources allocation to control interference. The paper [7-8] focuses on power control strategy to reduce interference. In order to improve the D2D communication performance and reduce the cellular interference, this paper introduces the network coding in D2D communication. Network coding was first proposes in paper [9] and then become a research focal point. Network coding refers to the ability of a node in the network to appropriated encode the incoming data before sending these coded the data at the intermediate nodes results in a substantial bandwidth improvement over that of a traditional store and forward networks. Network coding is that the relay node not just acts as a transmit node but also encodes the received information and then forward the new information to the destination.

The paper [10] first applied network coding into D2D communication. This paper introduces network coding to complete information exchange, like traditional two-way relay system in cellular network, but different in the D2D

$*$

This paper is supported by National Important Special Project [2012ZX3003011-005]; NSF funding of china[61171093]; Jiangsu Province Important Special

Project[BK2011027]; School Project[NY211033]. 
communication environment. The paper [11] describes the ordinary two-way relay performance and papers [12-13] analyze the performance with consideration of the noise.

The organization of this article is as follows: We first discussed the D2D network coding model in section 2.We analysis different scheme with and without network coding in section 3 . Section 4 presents the simulation results that confirm our theoretical and section 5 is conclusion.

\section{System Model}

The paper consider the D2D communication performance with network coding, the system model is shown in figure 1.In a single cell, there is a D2D pair which consists of node A and $\mathrm{B}$, this D2D pair shares resource with cellular user denoted by C-UE. All nodes are equipped with single antenna and work in half-duplex mode. In D2D mode node A and node B exchange information through relay node $\mathrm{R}$ and receive node will receive interference from C UE.

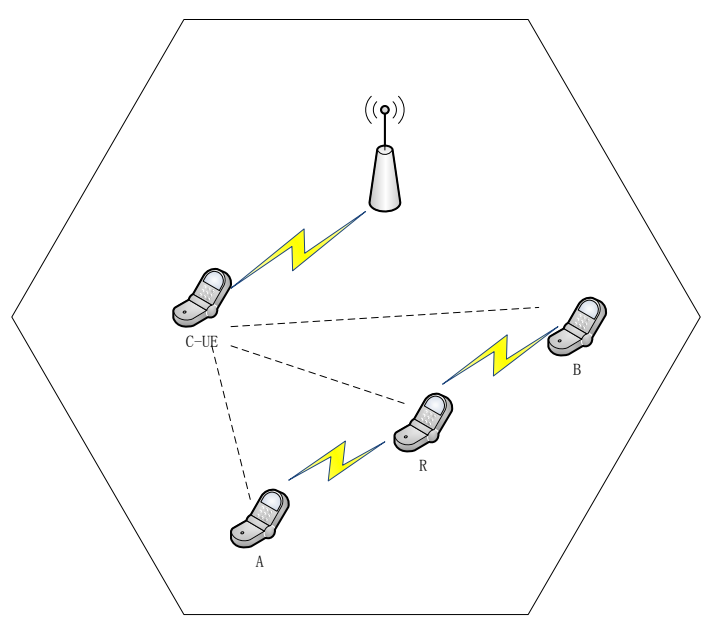

Figure 1.D2D network coding system

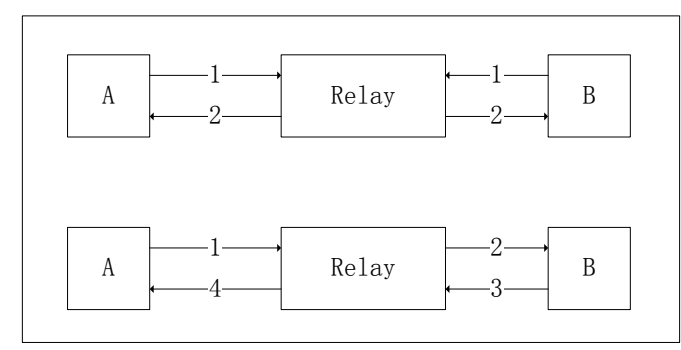

Figure 2.network coding transmission and traditional transmission

As shown in figure 2, the process of information exchange between $\mathrm{A}$ and $\mathrm{B}$ using network coding transmission requires two time slots. In the first time slot, node A and B both sent information to the relay $\mathrm{R}$, and then, the relay receives the data of two source terminals, the relay forms a new packet by XOR like $x_{r}=x_{1} \oplus x_{2}$. In the second time slot, relay $\mathrm{R}$ sends the new packet to both $\mathrm{A}$ and $\mathrm{B}$. The node $\mathrm{A}$ and $\mathrm{B}$ can decode the received information by self-information
$x_{A}{ }^{*}=x_{1} \oplus\left(x_{1} \oplus x_{2}\right)=x_{2}$. Because this $\mathrm{D} 2 \mathrm{D}$ pair is reuse resource with cell user C-UE, information transmitted in the above slots will be interfered by C-UE. But in the traditional transmission mode exchange information need four slots, the first slots node A transmits to the relay node and the second slot relay $\mathrm{R}$ forward data to node $\mathrm{B}$; the third slot node $\mathrm{B}$ transmits to the relay node and the fourth slot relay $\mathrm{R}$ forward data to the node A.

There may be one or more D2D pair reuse the same cellular user resource, so D2D communication will interference by other D2D pairs, we omit the analysis for this case.

\section{D2D network coding performance analyses}

We assume that there is no direct link between $\mathrm{A}$ and $\mathrm{B}$, and all the channel state information (CSI) is known. The transmission signal of $\mathrm{A}$ and $\mathrm{B}$ is $x_{A}$ and $x_{B}$ respectively, transmitting power $P_{A}=P_{B}=P_{R}=P$ and cellular user transmitting power is $P_{c}$. The paper wills comparison of the two different ways.

\subsection{Network coding forward mode}

In the first slot, node A and B simultaneously to the relay $\mathrm{R}$, the relay $\mathrm{R}$ not only receive node $\mathrm{A}$ and $\mathrm{B}$ data but also inference from $\mathrm{C}_{-} \mathrm{UE}$, so the signal received at relay $\mathrm{R}$ can be denoted by:

$$
y_{r}=h_{a r} x_{A}+h_{b r} x_{B}+h_{c r} x_{c}+n_{r}
$$

Where $h_{i j}$ is the channel state information between node $\mathrm{i}$ and j. $n_{r}$ is the Additive White Gaussian Noise (AWGN) at relay R with zero mean and average power equal $N_{0} \cdot x_{c}$ is the interference from cellular user what D2D pair share resource.

In the second slot, node A and B received the data can be denoted by:

$$
\begin{aligned}
& y_{A}=\mathrm{h}_{r a} \mathrm{x}_{r}+h_{c a} x_{c}+n_{A} \\
& y_{B}=\mathrm{h}_{r b} \mathrm{x}_{r}+h_{c b} x_{c}+n_{B}
\end{aligned}
$$

Where we assume that channel state information is symmetry and $h_{r a}=h_{a r} . n_{A}\left(n_{B}\right)$ is also the Additive White Gaussian Noise with zero mean and average power equal $N_{0}$.

The paper comparisons of the different forward way of relay both AF (amplify-and-forward) and DF (decode-andforward) mode to forward data. if we use AF scheme here, the signal $x_{r}$ is simply amplifies receiving signal $y_{r}$ and amplification gain is G. if we take DF mode, the transmitting signal $x_{r}$ is a new signal which contains information of $x_{A}$ and $x_{B}$. 
From figure 3, we can know that DF mode has a better performance than AF mode. As AF mode not only amplifies the signal but also the noise and interference between cellular user and D2D user that cause system performance degradation. Therefore we have adopted the DF mode to forward the signal. Then we can derive the received signal-to-noise (SNR) of $\mathrm{A}$ and $\mathrm{B}$ denoted by:

$$
r_{A}=\frac{\left|h_{r a}\right|^{2} P_{R}}{\left|h_{c a}\right|^{2} P_{c}+N_{0}}
$$

Similarly we also can know that node B receives SNR is:

$$
r_{B}=\frac{\left|h_{r b}\right|^{2} P_{R}}{\left|h_{c b}\right|^{2} P_{c}+N_{0}}
$$

\subsection{Traditional forward mode}

We can conclude that the reception signal of the node A and $\mathrm{B}$ in the traditional forward mode:

$$
\begin{aligned}
& y_{A_{-} t}=h_{r a} x_{b}+h_{c a} x_{c}+n_{A} \\
& y_{B_{-} t}=h_{r b} x_{a}+h_{c b} x_{c}+n_{B}
\end{aligned}
$$

We can know that the SNR of node $\mathrm{A}$ and $\mathrm{B}$ in the traditional mode:

$$
\begin{aligned}
& r_{A_{-} t}=\frac{\left|h_{r a}\right|^{2} P_{R}}{\left|h_{c a}\right|^{2} P_{C}+N_{0}} \\
& r_{B_{-} t}=\frac{\left|h_{r b}\right|^{2} P_{R}}{\left|h_{c b}\right|^{2} P_{C}+N_{0}}
\end{aligned}
$$

We assume that the total power is the same, so the relay $\mathrm{R}$ in the second and the fourth slots in the traditional forward mode transmit power is $\frac{P}{2}$.

We can conclude the system throughput as shown in figure 4 , the throughput of the system using network coding mode is higher than traditional mode. This is because using network coding can reduce slots for exchange information between node $\mathrm{A}$ and node $\mathrm{B}$, improve the efficiency of the system, so that increase the system throughput.

Next we comparison the two different mode impact of system outage probability, and in order to facilitate the calculation we assume that

$$
X_{1}=\frac{\left|h_{r a}\right|^{2} P_{R}}{N_{0}} \quad Y_{1}=\frac{\left|h_{c a}\right|^{2} P_{c}}{N_{0}}
$$

And $P_{R}=P_{C}=P$, so we can write the received SNR of A denoted by:

$$
r_{A}=\frac{\left|h_{r a}\right|^{2} P_{R}}{\left|h_{c a}\right|^{2} P_{c}+N_{0}}=\frac{X_{1}}{Y_{1}+1}
$$

In order to analyze the system performance, we assume that the threshold is $\mathrm{R}$ and system interrupt if either node of A or B is lower than R. Due to the distribution of $A$ and $B$ are independent, the outage probability can be written as:

$$
\begin{aligned}
P_{\text {out }} & =P\left(r_{A}<\operatorname{Rorr}_{B}<R\right) \\
& =1-P\left(r_{A}>R, r_{B}>R\right) \\
& =1-P\left(r_{A}>R\right) P\left(r_{B}>R\right)
\end{aligned}
$$

With the formula (11), we can know that the system outage probability can be simplified to calculate without interrupting the probability of A and B.

$$
\begin{aligned}
P\left(r_{A}>R\right) & =P\left(\frac{X_{1}}{Y_{1}+1}>R\right) \\
& =P\left(X_{1}>R\left(Y_{1}+1\right)\right) \\
& =\int P\left(X_{1}>R(R+1)\right) f_{y_{1}}\left(y_{1}\right) d y
\end{aligned}
$$

Because $X_{1}$ and $Y_{1}$ are independent and identically distributed, they are obey random variable $\chi^{2}$ distribution and their degree of freedom is $2 M^{2}$. So the probability density function (PDF) of $\mathrm{X}$ and $\mathrm{Y}$ can be written as:

$$
\begin{aligned}
& f_{x_{1}}\left(x_{1}\right)=\frac{1}{2 r_{1}} \exp \left(-\frac{x_{1}}{2 r_{1}}\right) \\
& f_{y_{1}}\left(y_{1}\right)=\frac{1}{2 r_{c}} \exp \left(-\frac{y_{1}}{2 r_{c}}\right) \\
& \text { Which } \mathrm{r}_{1}=\frac{P_{R}}{N_{0}} \Omega, \mathrm{r}_{c}=\frac{P_{c}}{N_{0}} \Omega \text { and } \Omega \text { is path loss. }
\end{aligned}
$$

$\Omega=d^{-v}$ And $V=-4$. So we can conclude that: 


$$
\begin{aligned}
& P\left(r_{A}>R\right) \\
& =\int_{0}^{\infty} f_{y_{1}}\left(y_{1}\right) d y_{1} \int_{0}^{R\left(Y_{1}+1\right)} \frac{1}{2 r_{1}} \exp \left(-\frac{x_{1}}{2 r_{1}}\right) d x \\
& =\int_{0}^{\infty} \exp \left(-\frac{R\left(y_{1}+1\right)}{2 r_{1}}\right) \frac{1}{2 r_{c}} \exp \left(-\frac{y}{2 r_{c}}\right) d y_{1} \\
& =\frac{r_{1}}{R r_{c}+r_{1}} \exp \left(-\frac{R}{2 r_{1}}\right)
\end{aligned}
$$

Similarly the node B can be written as:

$$
P\left(r_{B}>R\right)=\frac{r_{2}}{R r_{c}+r_{2}} \exp \left(-\frac{R}{2 r_{2}}\right)
$$

Take (15) and (16) into (11), we can derive the final system outage probability.

\section{Simulation result and analysis}

We use MATLAB simulation tool to demonstrate the correctness of our formula. Simulation environment is a single cell and the distance is normalized. The distance between node $\mathrm{A}(\mathrm{B})$ and the relay $\mathrm{R}$ is $d_{1}\left(d_{2}\right)$, and the distance between $\mathrm{C}$ $\mathrm{UE}$ and the D2D pair source is $d_{c}$. System outage probability as the SNR curves as figure 5 shown.

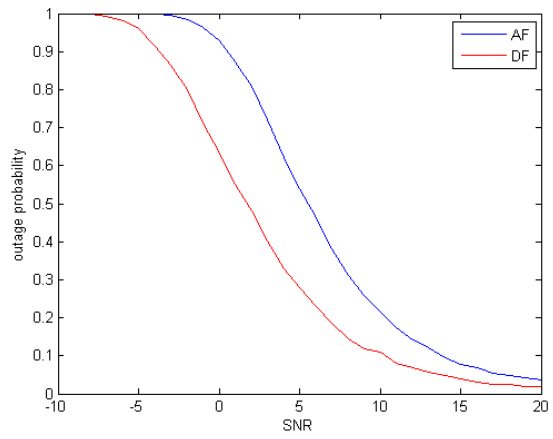

Figure 3.AF and DF mode performance simulation

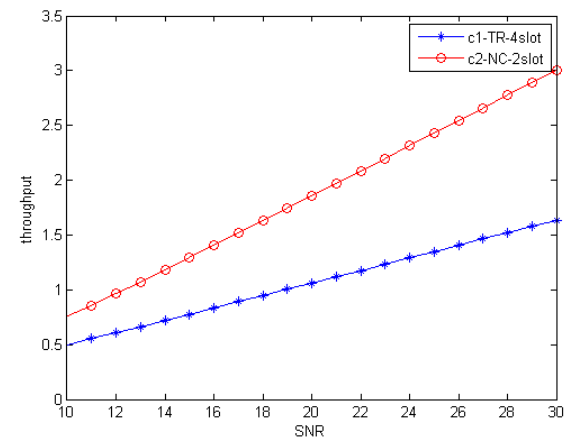

Figure 4.Network coding transmission throughput and traditional transmission throughput

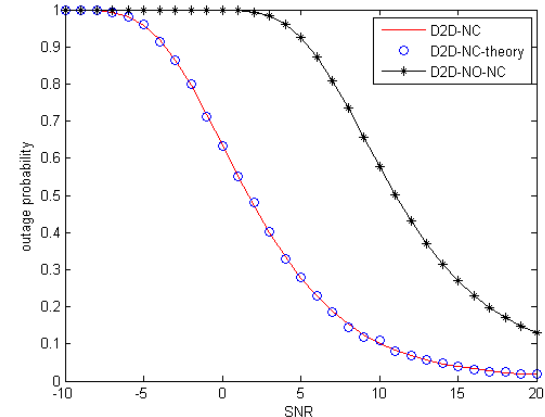

Figure 5. Performance comparison (with and without network coding)

System SNR shown in figure 3 varies from -10 to $20 \mathrm{~dB}$. From the figure we can know that the theoretical performance of D2D communication under network coding techniques conforms to the practice, the correctness of the derived formula in this article is verified. Compare to the D2D communication without network coding, we can see that the proposed method greatly reduces the system outage probability and increase the stability of the system.

Here we investigate the power allocation problem, how to ensure the normal communication at the same time to reduce the power consumption. We introduce the concept of allocation coefficients $\varepsilon=\frac{P_{R}}{P_{C}}$, from figure 6 , we can see that with the increase of allocation coefficient $\varepsilon$ system outage probability decreases.

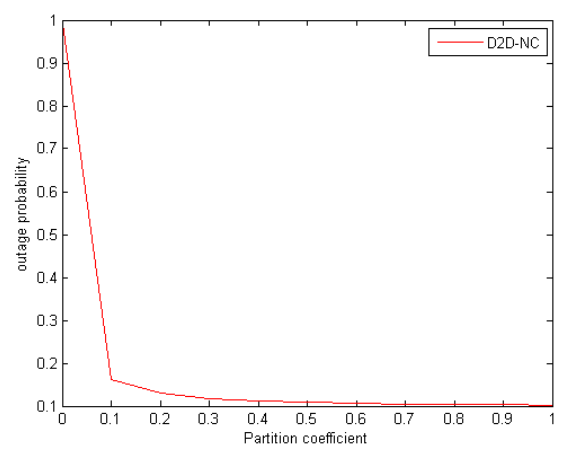

Figure 6. Allocation coefficient

As we can see from the figure 6 , the system probability falls vigorously when the allocation coefficient lies between 0 and 0.1 . While in the 0.1 to 1 the performance is almost the same. Then we can infer that the system achieves its best performance when $\varepsilon=\frac{P}{P_{c}}=0.1$. From (12) we can know the outage probability can be simplified to

$$
P\left(r_{A}>R\right)=K * \frac{1}{\frac{R}{\varepsilon}\left(\frac{d_{c}}{d_{1}}\right)^{-v}+1} .
$$


From (17), we can see that when $\varepsilon$ lies between 0 and

$0.1, P\left(r_{A}>R\right)$ will be change with the different $\varepsilon$. However

$P\left(r_{A}>R\right)$ tend to a fixed value when $\varepsilon$ varies from 0.1 to 1 , which conforms to figure 6 and verify the correctness of our conclusion.

\section{Conclusions}

As a research of hotspot of next generation communication, D2D technology as traditional cellular communication assists communication. The paper presented a transmission scheme exploiting network coding as a support for direct D2D communication. A detail describes the D2D communication system in the cellular network at the presence of cellular interference and analyzes the performance of the system after the introduction of network coding. The proposed scheme was demonstrate through MATLAB simulation; In these simulations two modes with or without network coding were compared. The result of those simulation show that D2D communication with network coding has a better performance than D2D without network coding. At the last, we propose a simple power allocation method.

\section{Reference}

[1] P.Janis, C.H.-Yu, K.Doppler, C.Ribeiro, C.Wijting, K.Hug, Tirkkonen and V.Koivunen "Device-to-device communication underlaying cellular communications systems" [C] International Journal of Communications, Network and System Sciences, vol. 2, no. 3, pp. 169-178,2009

[2] K.Doppler, MP.Rinne, C.Wijting, C.Ribeiro and KHugl, "Device-to-device communication as an underlay to LTE-advanced networks" [C] IEEE Communniction. vol. 47 no. 12, pp. 42-49, 2009.
[3] Doppler.K, Rinne.M, P,Janis. P,Ribeiro, Hugl.K "Device-to-Device communication Functional Prospects for LTE-Advanced Networks" Communicational Workshops, 2009,ICC workshop 2009.IEEE International Conference pp.1-6,2009.

[4] C.H-Yu, K.Doppler, C.Ribeiro, O.Tirkkonen and K. Hugl "Performance impact of fading interference to device-to-device communication underplaying cellular networks," in Proc. IEEE PIMRC, pp. 858-862, Sep.pp.858-862,2009.

[5] Chia-Hao.Yu, Klaus.Doppler, Cassio.B and Olav Trikonen "Resource Sharing Optimization for Device-to-Device Communication Underlying Cellular Networks" Wireless Communication, IEEE Transactions pp.27522763, 2011.

[6] Zulhasnine.M, Changcheng.Huang, Srinivasan, "A Efficient resource allocation for device-to-device communication underlaying LTE network" $[\mathrm{C}]$ Wireless and Mobile Computing, Networking and Communications (WiMob), pp.368-375, 2010.

[7] C.-H.Yu, K.Doppler, C. Ribeiro, and O. Tirkkonen "Power optimization of device-to-device communication underlaying cellular communication systems" IEEE International Conference on Communications pp.1-5,2009.

[8] Jaheon.Gu, Sueng.Jae.Bae, BumGon.Choi, Min-Young.Chung; "Dynamic power control mechanism for interference coordination of device-to-device communication in cellular networks"[C] Ubiquitous and Future Networks (ICUFN), pp.71-75, 2011.

[9] R.Alshwede, N.Cai, R.W. Yeung "Network information flow" IEEE Transaction, pp. 1204-1216, 2000.

[10] Rodziewicz, Marcin "Network coding aided Device-to-Device communication "European Wireless conference pp.1-5, 2012.

[11] Yonghu Hu, Kwok Hung, LiKah Chan "The Performance of two-way amplify-and-forward relay networks over asymmetric channels "Military Communication Conference pp.1-6,2009.

[12] Benevides.da, Costa,D, Haiyang.Ding, Jianhua.Ge "Interferfnce-Limited Relaying Transmissions in Dual-hop Cooperative Networks over Nakagami-m Fading" Communications Letters IEEE pp.503-505,2011.

[13] Caijun.Zhong, Shi Jin, Kai-kit Wong "Dual-hop system with noisy relay and interference-limited destination" Communication IEEE transactions pp.764-768, 2010. 